\title{
Loss of MLH1 regulates a metabolic phenotype in endometrial cancer
}

Endometrial cancer is the fourth most common cancer in women and the most common gynaecological malignancy in the developed world. No new systemic treatments for endometrial cancer have been developed in recent years and its incidence is expected to double over the next decade. As such, there is a need to gain a better understanding of key molecular pathways that are altered in the disease and could be targeted by novel treatments. The DNA mismatch repair (MMR) pathway is lost in approximately $30 \%$ of endometrial cancers. Recently, our lab has shown that MLH1deficient cells demonstrate a mitochondrial phenotype characterised by reduced oxidative phosphorylation (OXPHOS), reduced mtDNA copy number and Complex I inhibition. OXPHOS-deficient cells have to adapt their metabolism to compensate for energy defects and the inability to efficiently use the tricarboxylic acid cycle to generate energy. We hypothesise that this altered metabolism is driving tumourigenesis by increasing the metastatic potential of the tumour cells and are investigating this using orthotopic models of MLH1-deficient endometrial cancer in vivo. We have performed metabolomic analysis on a panel of MLH1-proficient and deficient paired endometrial cell lines and identified a metabolic map of alterations upon MLH1 loss. Ultimately, we aim to use this knowledge of altered metabolism upon MLH1 loss to identify more targeted treatments for MMR-deficient endometrial cancer patients. 